\title{
reCHERches
}

Culture et histoire dans l'espace roman

4 | 2010

Les représentations du corps dans la litterature latinoaméricaine

\section{Médiatisation et mythification du corps dans Un ojo llamado cacería de Marcela Saldaño}

Nouvelle poésie d'avant-garde chilienne

\section{Cécile-Alice Jouannaux}

\section{OpenEdition \\ Journals}

Édition électronique

URL : https://journals.openedition.org/cher/8300

DOI : $10.4000 /$ cher.8300

ISSN : 2803-5992

\section{Éditeur}

Presses universitaires de Strasbourg

\section{Édition imprimée}

Date de publication : 30 juin 2010

Pagination : 205-214

ISBN : 978-2-35410-007-0

ISSN : $1968-035 X$

\section{Référence électronique}

Cécile-Alice Jouannaux, « Médiatisation et mythification du corps dans Un ojo llamado cacería de Marcela Saldaño », reCHERches [En ligne], 4 | 2010, mis en ligne le 15 décembre 2021, consulté le 25 janvier 2022. URL : http://journals.openedition.org/cher/8300 ; DOI : https://doi.org/10.4000/cher. 8300

\section{(c) (†) $(9)$}

Ce(tte) œuvre est mise à disposition selon les termes de la Licence Creative Commons Attribution Pas d'Utilisation Commerciale - Partage dans les Mêmes Conditions 4.0 International. 


\title{
Médiatisation et mythification du corps dans Un ojo llamado cacería de Marcela Saldaño Nouvelle poésie d'avant-garde chilienne
}

\author{
CéCILE-Alice Jouannaux \\ Université Paris III - Sorbonne Nouvelle
}

\begin{abstract}
T e corps est, selon Émile Littré, «[ce] qui fait l'existence matérielle d'un Lhomme [...], vivant ou mort» (Littré 1874: 816), c'est-à-dire ce qui atteste physiquement de l'existence, donc de la présence d'un individu. Lien avec l'autre et les autres, son écriture est un thème commun à toute la production poétique de la nouvelle avant-garde chilienne, qui joue avec les codes sociaux et se défait des conventions, en usant de tous les ressorts de la provocation.
\end{abstract}

Dans son recueil de poèmes intitulé Un ojo llamado cacería, Marcela Saldaño ${ }^{1}$ ne se contente pas de mettre en vers le corps, elle le problématise, en en faisant le lieu du Paradoxe, celui de la «cacería»/ «carnicería ${ }^{2}$. Elle ne sombre pas non plus dans l'exploitation de ce que Sylvain Floc'h

1 Marcela Saldaño, née en 1981 à Santiago du Chili, participe activement des activités poétiques de Descentralización poética, organisation qui porte la Poésie en dehors des lieux qui lui sont habituellement consacrés, la rendant ainsi accessible à un large public. Le site Internet de Descentralización poética peut être consulté à l'adresse suivante: URL: http://descentralizacionpoetica.blogspot.com

2 Ces termes sont ingénieusement choisis car la «carnicería» contient en son sein la «cacería». 
appelle «l'obscénité culturelle-référentielle», en préservant le caractère «insaisissable» de la révolution qu'elle entend mener ${ }^{3}$.

Cet article démontre que l'œil acquiert une toute-puissance et faiblesse parce qu'il porte en lui la synthèse de tous les paradoxes. Puis, il met en évidence comment, de la même manière que la poétesse déjoue les écueils de la mise en vers conventionnelle du corps dans son entièreté, elle compose et décompose les matériaux de la phrase, pour donner un «corps» nouveau au langage poétique. Et à ce don, à cette offrande, inscrivant le recueil dans le registre du sacré, du sacrifice, s'associent les thèmes du Temps et les éléments de la légende dont l'analyse est indispensable pour comprendre la construction du mythe saldanien.

\section{L'œil, synthèse des paradoxes dans Un ojo llamado cacería}

Le corps, parce qu'il est ce que perçoivent immédiatement les autres d'un individu, devient à la fois signifiant et signifié. Porteur de sens, le corps active une dialectique complexe. Les termes «brisé » et "caducité», présents dans la description des enjeux de la représentation du corps de Gérard Durozoi ${ }^{4}$ renvoient précisément à la brèche dans laquelle s'engouffre Marcela Saldaño en mettant en exergue l'œil, organe cyclopéen devenant obscène à force d'être omniprésent dans son recueil.

Apparaissant dès le titre de l'œuvre Un ojo llamado cacería, l'œil est l'emblème du Paradoxe, clef de la compréhension du recueil car il est l'organe qui permet le premier contact avec le monde et la rencontre avec notre reflet dans le miroir. De cette action spéculaire naît le premier paradoxe du recueil (l'œil qui voit est vu par lui-même et les autres qui le voient) mis en valeur dans le titre Un ojo llamado cacería car l'œil chasseur est immanquablement chassé en un renversement de situation sans fin: «El

3 Citation in extenso de Sylvain Floc'h: «Si l'obscénité culturelle-référentielle se cantonne au domaine du choquant, de l'indécent, de l'anticonformisme, en un mot de l'envers des valeurs, la véritable obscénité, en revanche, ne se laisse pas si facilement épinglée au revers des convenances. Elle ne joue jamais l'envers du code, elle joue sa propre réversibilité. Elle vit ses orgies et ses bacchanales comme rébellion radicale (c'est d'ailleurs le sens étymologique du verbe anglais «to revel», dérivé de «rebelle»). En déjouant ainsi l'envers et l'endroit, le révoltant rend insaisissable sa révolte» (en gras dans le texte) (Floc'h 1983:13).

4 «Ce qui est représenté est moins le corps dans sa réalité que les conceptions qu'en privilégie une époque, une société, une morale; briser cette représentation, c'est donc révéler la caducité de ces conceptions» (Durozoi 2005: 40) 
ojo grita Tiembla en medio de las bestias que lo devoran Aunque ellas no saben que son las presas $»^{5}$.

Dans une des réponses à notre questionnaire ${ }^{6}$, Marcela Saldaño décrit le travail insatiable de cet œil dans son recueil:

Es el ojo cazador, el ojo carnicero, el ojo que todo lo captura, pero en su captura mata todo también, el ojo que espera, el ojo que también pueden ser los agujeros del cuerpo y captar la luz, dar a luz, sacar desperdicios, oír, oler, probar, degustar, expulsar, la arcada, la saliva, el desperdicio, [...] el ojo también puede convertirse en la esfera semiológica no obstante la cacería es el martillo con el que se llega a la carnicería la idea de mi obra es romper con esa esfera y crear un imaginario donde brille el ojo como un ente que muchas veces es utilizado sólo como objeto [...].

[...] La forma que el ojo cazador adquiere, que es el de la carnicería, carnicería semiológica, la cacería de crear una herida visible e ignorada y sanarla en la consciencia de esa herida [...]. (Saldaño: 16/02/09)

Le paradoxe du recueil réside aussi dans le choix du titre et de l'illustration de la page de couverture réalisée par le dessinateur italien Antonio Vitale. De façon surprenante, le dessin qui accompagne le titre représente un visage sans yeux. Les lèvres de la femme dessinée sont très charnues, ses dents ne sont pas visibles, et ce qu'elle maintient avec force est un poisson et non de la viande, qui aurait été en adéquation avec le thème de la boucherie développé dans le recueil. Et au final, les yeux (ou l'«œil»), qui manque(nt) à cette femme, ce sont les lecteurs qui le(s) possèdent en voyant la représentation de ce demi-visage de femme. Apparaît ici à nouveau le jeu de reflet évoqué précédemment, ainsi que la coexistence des contraires (eau / feu, absence de la vue / hyperlucidité) que permet la poétesse-démiurge, manifestation de la «réversibilité» (Floc'h 1983: 13) de ses propres codes / anti-codes:

Esta paradoja es justamente el sentido de la obra, es la carnicería semiológica, elegir el pescado como presa lleva consigo el poder del ojo de capturar lo que desee y llevar la sangre a lo sin sangre, generar la carne en donde sólo estamos hablando de la sustancia muerta [...]. Debido a est[a] [imagen] también se recrea el poema donde digo: "tu cuerpo líquido esquiva mi mordida", el agua adquiere el valor del fuego y el pez adquiere también el brillo de la ceguera de esa mujer, la ceguera que es videncia en el libro un libro vidente que es ciego en el dibujo y caza un pez en vez de un animal, no obstante el primero en cazarse es el propio ojo en su nuevo nombre que es cacería.

5 Extrait du poème «El ojo de los oscuros papeles» ouvrant le recueil (p. 11).

6 Cet article est enrichi de l'analyse des réponses de Marcela Saldaño à nos questionnairescommentaires sur son œuvre. 
L'œil «regardant/ boucher»et «regardé/ chassé» entretient dans les poèmes de Marcela Saldaño des relations étroites avec la chair qui pourrait paraître déconcertante mais qui, en réalité, ne sont que la systématisation des actions que l'on attribue habituellement à l'œil. En effet, dans le langage courant les expressions relevant de la mise à mort telles que «fulminar a alguien con la mirada» («fusiller quelqu'un du regard») ou "devorar con la mirada» ("dévorer quelqu'un des yeux») sont nombreuses.

Dans le recueil Un ojo llamado cacería, le thème de la «boucherie», qui évoque l'acte de couper et suggère la consommation de la chair, crée une synesthésie entre le «toucher» et le «voir». La boucherie devient action et résultat de cette action que l'œil est capable de réaliser, de la même manière qu'il est à la fois chasseur et chassé. Elle est exprimée par la récurrence des objets tranchants dans les poèmes de Marcela Saldaño, et est de manière paradoxale, sans pitié, selon le second sens de ce même terme qui convoque l'imaginaire du déferlement de violence sanguinaire, du massacre.

Ces paradoxes ne sont que quelques-uns des maillons de cette chaîne de paradoxes que régit l'aphorisme "el primer estado es agua» («le premier état est eau»). Si la poétesse est liée à la chair, à la chasse et à la boucherie, elle est aussi eau, tout comme l'œil. Et de manière analogique, ils sont tous deux reptiliens comme l'annonçait la transfiguration poétique de Marcela Saldaño en «MarSerpiente»?.

Le jeu avec les paradoxes auquel s'adonne la poétesse, s'accompagne d'un travail plus en amont car le poème, mise en vers de cette chasse et de cette boucherie, est le résultat de la corporification du langage.

\section{Corporification du langage et sacrifice}

L'emprunt d'un terme à la Théologie et à la Chimie, tel que celui de «corporification», pour éclairer le travail poétique de Marcela Saldaño qui restitue un caractère "sacré» à ce qui n'en a pas et réinvente les états de la matière, est particulièrement utile. Plus précisément les définitions du verbe "corporifier» et de ses dérivés, "corporification» et "corporéité », permettent de rendre compte de ce cheminement du sacré à la matière, et de la matière aux mots:

$7 \quad$ Il existe une analogie entre les deux: la fluidité de l'eau et du reptile matérialisée par la similitude phonétique [mar][se] (La fluidité des sonorités en [m] et les sifflantes [s] participent de la création d'un univers fluide, liquide). 
Corporifier: $1^{\circ}$ Terme de théologie. Supposer, donner un corps à ce qui est esprit. Les hérétiques qui corporifiaient les anges. $2^{\circ}$ Ancien terme de chimie. Fixer en corps les parties éparses d'une substance. Corporifier du mercure. (Littré: 816)

Corporification: Ancien terme de chimie. Action de condenser des vapeurs en un corps solide. (Littré: 816)

Corporéité: Terme didactique. Ce qui constitue un corps tel qu'il est. L'âme ne forme des idées spirituelles qu'à l'aide des mots qui en sont les signes, et ces mots prouvent la corporéité de ces idées ${ }^{8}$. (Littré: 816 )

Ainsi, si l'on se réfère à la définition de ces emprunts, la "corporification» est le résultat de l'action de "corporifier» qui signifie «donner un corps à ce qui n'en a pas», autrement dit le langage (en mots et en ponctuation), et ce d'autant plus que, dans Un ojo llamado cacería, le corps mis en poème est luimême atteint dans son intégrité, décomposé / recomposé en des segments de phrases pour signifier "autre chose». Cette analogie entre langage et corps est possible car selon Hans Bellmer (Anatomie de l'image, 1957), «Le corps est comparable à une phrase qui nous inviterait à la désarticuler, pour que se recomposent, à travers une série d'anagrammes sans fin, ses contenus véritables». (Durozoi: 40)

Mais comme dans le recueil de Marcela Saldaño, un rapport extrême avec le corps, la "carnicería », est mis en vers, une violence est faite au texte lui-même. Cette violence se remarque dans la présentation générale du recueil: de très nombreux espaces libres créent la respiration interne au sein de celui-ci alors qu'il n'y a pas de ponctuation dans les vers. Un vers occupe parfois à lui seul une page comme si le but était d'augmenter l'impact des mots qu'il contient, comme si celui-ci était éviscéré et étalé sur une table, la page blanche, qui peut être à la fois de dissection et de boucherie, regardé par l'œil, cet astre cyclope, ce démiurge toujours à la recherche de sa proie, une des représentations possibles de la poétesse, et par le lecteur également. Une des réponses de Marcela Saldaño à nos questions, citée ici in extenso, expose clairement le lien entre le choix de la forme du recueil et la «chasseboucherie» à l'œuvre dans celui-ci :

Dentro de la obra en general, el ojo se sirve de micro obras que son las que Ud observa, este es un libro que se puede leer de corrido y también de manera independiente, no obstante la falta de puntuación es una manera de cazar una regla y exterminarla, una carnicería más adentro del libro y respecto a las mayúsculas representan el corte del verso, incluso es más que eso, la mayúscula va en íntima relación con el ritmo del poema, es el corte según

8 «Bonnet, Charles, 1779-1788, Euvres, Berne, édit. citée par Pougens» (Littré: 2622). 
lectura e impulso musical, la mano musical el ojo que es imagen y música, además me permite lograr densidad, pequeños ladrillos que construyen la casa del ojo, la esfera de sangre que tiro contra la pared del imaginario que construyo, también la mayúscula es la mancha y el deseo, lo insaciable de la cacería-carnicera. (Saldaño: 18/02/2009)

L'action de donner à voir cet «imaginaire qu' [elle construit]» se rapporte à la notion de «communicabilité », extrêmement importante chez Marcela Saldaño, qui entretient des rapports étroits avec la pratique sacrificielle? ${ }^{9}$ Et un des vecteurs, qui rendent possible cette communication, est précisément l'œil mais aussi le sang, car de l'approfondissement de son travail poétique, résulte une intériorisation du corps, donnant lieu à une médiatisation grâce à des fragments ou à des substances émanant de celui-ci.

Le sang est un leitmotiv dans la création poétique de Marcela Saldaño. Il est un des éléments les plus malléables du corps. Dans sa poésie, il est celui grâce auquel le corps devient malléable. Le sang est aussi la preuve et le résultat de l'engagement total de Marcela Saldaño pour la poésie dont l'écriture répond à une "nécessité biologique»:

[...] [S]iempre [miro] hacia el lado de la moneda que está oculta, sólo de ese modo puedo volver a renacer en el fuego de la escritura y escribir con sangre, porque creo que sin sangre no hay poesía, considero que hay muchos poetas sin sangre y eso va en contra de mi carnicería, quiero artistas comprometidos, yo arriesgo mi vida por la poesía, es una necesidad biológica, la poesía me problematiza y me soluciona. (Saldaño: 18/02/2009)

De plus, il existe un lien indéfectible entre la poésie et le corps "décorporifié» («charco de sangre») que Marcela Saldaño expose dans une de ses réponses semblable à un art poétique:

[...] [M]i labor es escribir poesía para que pueda ser leída por el mundo y no explorar de manera publicitaria nichos, quiero una poesía universal, escribir para quien quiera leer, crear imaginarios que asesinen los conceptos anacrónicos de la creación que también pueden ser brillosos y sutiles al nadar o patalear en este charco de sangre que es bello como decía Aimé Césaire. Necesario es coser los agujeros del cuerpo y purificarlos para que sean de todos. Ese sacrificio que se resiste a la página en blanco y me resisto a dejar de resistir en la poesía que es la revolución primera y espero el desastre para marcharme y volver por otro. (Saldaño: 16/02/2008)

Le poème devient le lieu d'un sacrifice, il est offrande «à ceux qui veulent lire » avec une connotation sacrée appuyée mais pas religieuse au sens de réunion

9 Nicole Brenez, dans son ouvrage Cinéma d'avant-garde, compare l'action de l'artiste avant-gardiste, nécessairement engagé, à un «sacrifice» (Brenez 2006: 3-4; 7). 
«religare», car il n'y a paradoxalement pas de communion avec ces derniers et la poétesse, qui les laisse seuls face à la lecture de son œuvre. Comme le souligne Durozoi «communier ne renvoie plus à aucune transcendance: c'est une potentialité de la chair et des affects»(Durozoi: 42).

Plus encore, ce sacrifice fait partie intégrante du mythe qu'élabore au fil des poèmes Marcela Saldaño.

\section{Construction du mythe saldanien}

Ce sacrifice intimement associé à la récurrence du thème du Temps et le métadiscours sur la création poétique, point commun à tous les poètes d'avantgarde, sont liés au Hors-Temps (emploi de l'adjectif «anachronique»). Pour Marcela Saldaño, la Poésie est la «Révolution première»; ce qui suggère un recommencement, un cycle. Selon la même idée de retour, le poème, dont les antécédents sont expérimentaux, est la manifestation d'un désastre vu comme une promesse de renaissance pour la poétesse, inscrivant toujours davantage le recueil dans le registre du sacré. Selon Émile Littré, le désastre est:

Infortune très grande. [...] Le désastre est l'influence d'un astre qui cesse d'être favorable, c'est un malheur, un revers infligé par la fortune. La catastrophe est un renversement sens dessus dessous. La catastrophe est un désastre qui produit dans un ordre de choses, dans l'existence d'un individu, etc. un bouleversement complet ou une fin violente [...]. Il faut ajouter que la catastrophe est toujours instantanée ou à peu près, et qu'enfin elle peut être heureuse ou malheureuse, quoiqu'elle s'entende presque toujours dans ce dernier sens. (Littré: 1093).

Et cet astre bien présent dans ce recueil aux renversements permanents n'est autre que l'œil. Ainsi, l'œuvre poétique de Marcela Saldaño est à la fois son développement, sa finitude et son recommencement rendu possible par l'écriture.

Dans le contexte ambivalent de l'écriture de Marcela Saldaño, cette mythification du corps est rendue possible parce qu'elle remet en cause des contraires qui n'en sont pas en réalité: «el charco de sangre» est source de la création poétique et le "désastre», aussi, est productif, comme l'indique Émile Littré («désastre qui produit»). En réalité, c'est l'écriture même du corps qui génère l'union de ces contraires complémentaires et de tous les paradoxes. Selon Michel Journiac, cité par Durozoi, il faut: «Accepter 
comme origine existentielle, nécessaire de la création - lieu commun des contradictions: le corps, réel insurmontable ${ }^{10}$.

L'acceptation de cette précarité inhérente au thème du corps permettrait d'expliquer pourquoi rien n'est jamais donné pour acquis dans la poésie de Marcela Saldaño qui refuse de fournir des solutions définitives:

Que los otros busquen el antídoto, me vuelvo inmune en el libro. Y mi afán de cazar es insaciable y no me siento satisfecha, nunca nada me conforma. (Saldaño: 16/02/2008)

Marcela/ MarSerpiente, union improbable d'un élément liquide, la mer, et d'un autre à la fois terrestre et aquatique, est la déesse incarnant l'union des opposés: l'absence de corporéité véritable de l'eau et la corporéité du serpent. Mais la corporéité de cet animal malléable et toujours en mouvement, subissant des transformations cycliques, ses mues, fait écho aux renaissances de la poétesse, en même temps qu'elle rappelle que celle-ci poursuit perpétuellement sa «cacería», sa quête.

La transfiguration poétique de la poétesse en cet être hybride doué de pouvoirs, les thèmes de l'Hors-Temps et de la boucherie, appartiennent au mythe personnel de la poétesse. Le mythe n'est-il pas lié aux assassinats, aux sacrifices, au cannibalisme, à l'exacerbation des sensations, aux grands éléments? Dans les poèmes de Marcela Saldaño sont bien présents l'eau mais aussi le feu, le "gran ente», l'œil et la déesse, la MerSerpent qui prend vie dans ses poèmes:

Marserpiente, es el misterio en el que indago en mi poesía, pero no pretendo descubrir, circulo en el borde de este poema interminable que es mi producción por completo, necesito como poeta levantar el arma de la poesía a través de una mitificación pero propia, y que ésta esté en constante creación y circulación $[\ldots]$ y sin duda marserpiente desea salir y sale en las lecturas $[\ldots]$.

Dans ce mythe qui se construit de poèmes en poèmes, viennent également s'intégrer des éléments empruntés à la légende: la voyance associée à la cécité rappellant l'Antiquité Grecque et la sibylle, et les bois, lieux par excellence de l'irrationnel et de l'immonde dans les œuvres du Moyen-Âge.

En somme, Marcela Saldaño exploite les failles de l'écriture du corps en entreprenant dans son recueil un savant travail de décompositionrecomposition des éléments du mythe et de la légende, "se maintenant aux"

10 (Michel Journiac, 1972) cité dans (Durozoi : 162). 
et «se nourrissant des» limites de l'impossible (le Hors-Temps, l'hybridité de MarSerpiente).

\section{Conclusion}

De manière imagée, l'exploration du thème du corps démembré et liquéfié dans les poèmes de Un ojo llamado cacería est aussi abyssale que la mer à laquelle est intrinsèquement liée la MerSerpent.

Analyser les poèmes de Marcela Saldaño nécessite d'aller au-delà des paradoxes et contradictions apparents du corps et de s'introduire dans un nouveau champ de significations, fait de jeux subtils avec les analogies, de retours-recommencements, de références à d'autres cultures et époques, à la manière de la "spirale ${ }^{11}$, qui, pour Frankétienne, est une «structure absolue en perpétuel mouvement, [impliquant] une démarche globale où se retrouvent le social, $[\ldots]$ l'historique, $[\ldots]$ le collectif, $[\ldots]$ le religieux $[\ldots] »$.

\section{Bibliographie}

Ouvrages imprimés:

Brenez, Nicole, 2006, Cinéma d’avant-garde, Paris, Cahiers du Cinéma, coll. «Les petits cahiers «SCÉRÉN-CNDP».

Durozoi, Gérard, 2005, Dada et les arts rebelles. Les concepts. Les mouvements. Les artistes, Paris, Éditions Hazan, coll. «Guide des Arts».

Floc'h, Sylvain, 1983, «L'obscène et le révoltant», dans L'obscène (actes du colloque), Cahiers de l'Université $n^{\circ} 4$, Université de Pau et des Pays de l'Adour, Groupe de Recherche en Sociologie de la Littérature.

Littré, Émile, 1874, Dictionnaire de la langue française, Paris, Librairie Hachette et Compagnie.

Pane, Gina, 1973, «Le corps et son support-image pour une communication nonlinguistique», arTitudes international, $\mathrm{n}^{\circ} 3$, février-mars 1973 cité dans Durozoi, Gérard, 2005, Dada et les arts rebelles. Les concepts. Les mouvements. Les artistes, Paris, Éditions Hazan, coll. «Guide des Arts», p. 166-167.

Ressources électroniques:

Kaykayen, disponible sur Internet à l'adresse: URL: http://kaykayen.blogia.com, consulté le 07/02/09.

11 Le terme «spirale» est emprunté à Frankétienne et à Jean-Claude Fignolé. 
Potomitan. Site de promotion des cultures et des langues créoles, «Le dire du spiralisme: Entretien avec Frankétienne et Jean-Claude Fignolé», disponible à l'adresse: http://www.potomitan.info/colloques/fignole.php, consulté le 21/04/2009.

Saldaño, Marcela, 2008, Un ojo llamado cacería, Editorial Piedra del Sol, Santiago, Chili.

Saldaño, Marcela, 2008, «Respuestas», message électronique reçu le 16/02/2008.

Saldaño, Marcela, «Respuestas», message électronique reçu le 22/03/2009.

Saldaño, Marcela, 2009, http://marserpiente.blogspot.com/2009/01/PRESENTACINUN-OJO-LLAMADO-CACERA.htm ${ }^{12}$.

12 Avec les omissions de voyelles dans l'adresse. 